\title{
Tippy the Telepresence Robot
}

\author{
Johnty Wang, Vincent Tsao, Sidney Fels, and Benny Chan \\ Media and Graphics Interdisciplinary Centre \\ University of British Columbia \\ Vancouver, BC, Canada \\ \{johnty, vtsao,ssfels\}@ece.ubc.ca, bennyccy@gmail.com
}

\begin{abstract}
We have built Tippy, a micro telepresence robot that allows a user to remotely project him or herself into another space, move around, and communicate via video and audio. An iPod Touch running Skype provides the robot with wireless video input and output capabilities and handles the movement commands from the remote user. In order to handle the robot's movement without implementing an additional medium, we inserted the control signals visually into the video and detect the signals via light sensors connected to the motor drive system. By optically coupling the mobile device display with the robot's drive-train control, we reduce the amount of software and hardware interfacing required for the end application.
\end{abstract}

Keywords: telepresence, robot, optic coupling, hardware interfaces, human computer interaction, mobile applications.

\section{Introduction}

With increasingly powerful mobile devices, a significant amount of processing power is available to portable interactive applications. Numerous existing consumer level accessories such as keyboards, speakers, headsets and other add-ons extend the functionality of devices by providing extra interfaces. However, there is considerable development overhead when trying to interface mobile devices with new hardware. This paper presents Tippy (Fig. 1), a telepresence robot as an example application, which requires a hardware interface between mobile device and custom circuitry and describes the process of implementing a simple optically coupled interface to achieve this.

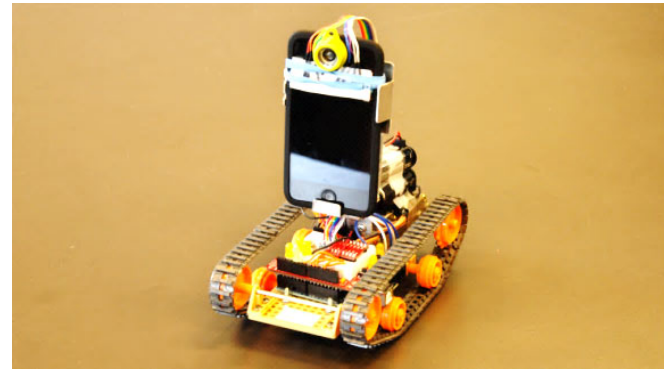

Fig. 1. Tippy the telepresensce robot 


\section{Motivation}

Two way video conferencing is now available on devices such as the iPod Touch [1]. The compact form factor and wireless capability of the iTouch make it an ideal platform for constructing an inexpensive telepresence [2] robot similar in functionality to [3], but in a smaller package. There are two ways that the iTouch based robot can be controlled remotely. First, the iTouch can be interfaced directly with the motor drive hardware using an iOS application that communicates with the hardware serial port. The application would have to run in background while the video application is active. Second, a dedicated, separate wireless link (using ZigBee, Bluetooth, or other Radio Frequency technologies) can be implemented on the robot to receive the remote commands and transfer them to the drive hardware.

The first option above is similar to [3], but it is considerably more difficult to directly interface with the iTouch's hardware port. The second option requires extra wireless hardware which adds considerably to the cost of the system. We found an alternative solution through overlaying the control signals directly into the video, and then building an optically coupled hardware interface between the iTouch and the motor control.

\section{Existing Work}

The concept of optical coupling using a display source is demonstrated in [4] where the average brightness value of a displayed character on a monitor is used to drive audio synthesis and in [5] where a mobile display is used to generate and display a sequence of codes to be transferred to another terminal. The difference between Tippy and these systems is that the optical signal is generated remotely and added as an overlay on top the incoming video signal. This way, the mobile device simply renders the video on screen as it is received and is not aware of the existence of the extra information. Because of this, any device with a front facing camera can be used in place of the iTouch with no additional modifications. Light sensors mounted on the screen detect the overlay signals and a microcontroller translates them into motor drive commands. As a teleconference robot, Tippy is significantly more compact compared to [3] and considerably cheaper ( $\$ 350$ vs. $\sim \$ 500)$.

\section{The Tippy System}

\subsection{Overview}

There are two main components of Tippy: the remote user control end and the robot end. The two parts are connected through the Internet via two-way video using Skype, as shown in Fig. 2. 


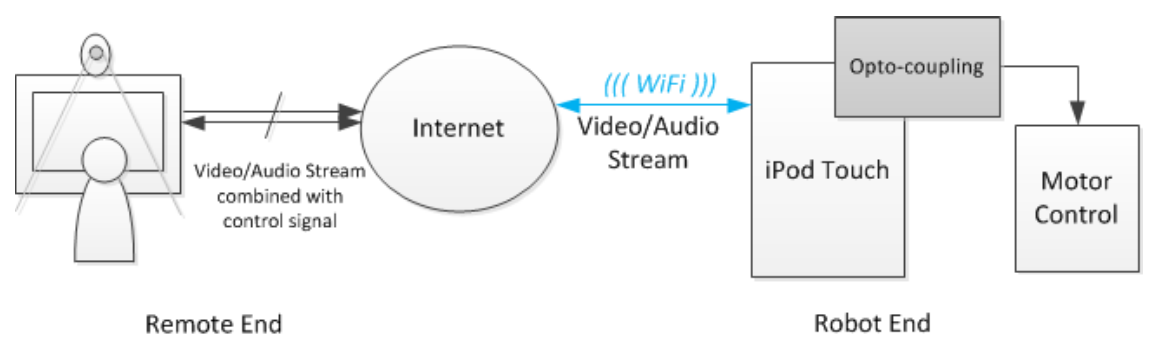

Fig. 2. Tippy system diagram

\subsection{Remote User End}

For normal two-way video conversation, Skype captures the video stream from a local input capture source (e.g.: a webcam) and transmits it to the remote end. For the Tippy system, in addition to the feed from the capture source, we also send the movement commands through the video. This is achieved through a custom Microsoft DirectShow filter that adds an overlay to the input source. The overlay consists of a black bar that appears at the top of the video image, with four white squares that light up in predefined places depending on the input command.

Using a binary representation with the four squares, up to sixteen discrete commands can be implemented (the current Tippy prototype only uses five). A simple GUI application was written to trigger each command, and the keyboard keys "w, a, s, and d" are mapped to forward, left, back, and right, respectively.

\subsection{Robot End}

On the robot end, the iTouch displays the video containing the overlay as it is received from Skype. A row of four photo-resistors are mounted on the LCD screen where the overlay appears. The photo-resistors are connected in a voltage divider configuration and the changing voltage is detected by an Arduino [6] microcontroller. Each sensor value is thresholded to produce a binary result. Programmed logic on the microcontroller drives the input pins of an integrated H-bridge motor driver, as shown in Fig. 3.

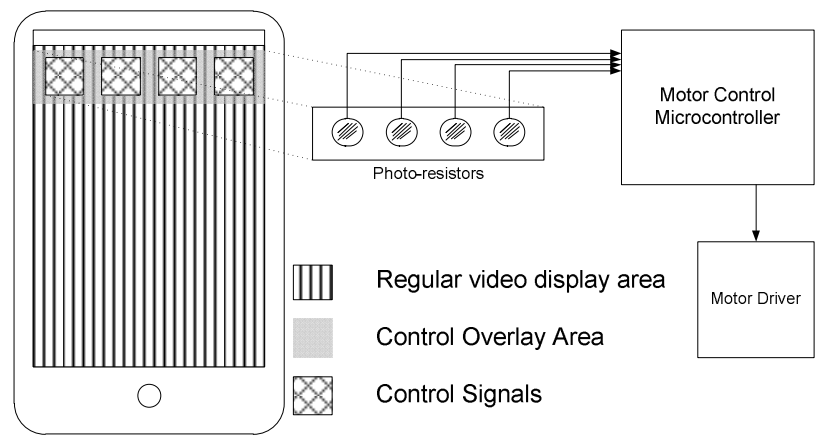

Fig. 3. The Tippy optical hardware interface 
To quickly prototype a working system, we used components from the Tamiya [7] wheel and track kit that provide a simple and flexible drivetrain platform. A dual channel motor driver provides bi-directional control of the geared motors driving each track and allows the full spectrum of motion afforded by a tracked vehicle.

\section{Future Development}

In the first prototype we only implemented four directions of movement. It would be quite easy to extend the number of discrete controls up to sixteen given the current optical hardware and software overlay. More complex movements can be implemented and variable speed control can be added using pulse width modulation control. For the optical interface, it would be possible to add a modulation scheme to provide a larger range of input values.

\section{Conclusion}

The concept of optic coupling can be used to easily extend the functionality of mobile devices as demonstrated through this project. Using mostly existing hardware and software we were able to quickly implement a low cost telepresence robot with little development overhead. We hope that the methodology employed can be used by others to rapidly prototype and demonstrate new and exciting ideas using mobile devices.

\section{References}

1. Apple Corporation, http: / /www . apple.com

2. Sheridan, T.B.: Musings on Telepresence and Virtual Presence. In: Proceedings from SRI's 1991 Conference on Virtual Reality on Virtual Worlds, Westport (1992)

3. Procrastineering,

http://procrastineering.blogspot.com/2011/02/

low-cost-video-chat-robot.html

4. Bianchi, A.: Authentication on Public Terminals with Private Devices. In: Proceedings of the Fifth International Conference on Tangible, Embedded, and Embodied Interaction, New York (2011)

5. McMullen, S.C., Winkler, F.: The Elocuter: I Must Remind You We Live in Dada Times. In: Proceedings of the 28th of the International Conference Extended Abstracts on Human Factors in Computing Systems (2010)

6. Arduino, http://www.arduino.cc

7. Tamiya, http://www.tamiya.com 